\title{
Bayesian estimation for the Kumaraswamy-inverse Rayleigh distribution based on progressive first failure censored samples
}

\author{
Reza Azimi* and Faramarz Azimi Sarikhanbaglu \\ Department of Statistics, Parsabad Moghan Branch, Islamic Azad University, Parsabad Moghan, Iran \\ *Corresponding author E-mail: azimireza1365@gmail.com
}

Copyright (C2014 Azimi et. al. This is an open access article distributed under the Creative Commons Attribution License Creative Commons Attribution License, which permits unrestricted use, distribution, and reproduction in any medium, provided the original work is properly cited.

\begin{abstract}
This paper considers Bayesian estimation of parameter and reliability function of Kumaraswamy-inverse Rayleigh distribution under the different loss functions with progressively first failure censored samples. We used squared error , minimum expected, weighted and Linex loss functions for obtaining the Bayes estimators of parameter and reliability function. Finally, Comparisons are made between Bayes estimators under different loss functions using simulation study.
\end{abstract}

Keywords: Kumaraswamy-inverse Rayleigh distribution, Progressive first failure censoring, Bayesian estimation, reliability function.

\section{Introduction}

The Kumaraswamy-inverse Weibull distribution was introduced by Shahbaz et. al [5]. This distribution is an extension of the Inverse Weibull distribution. The Kumaraswamy-inverse Weibull distribution has a cumulative distribution function of the form

$F(x)=1-\left[1-\exp \left(-\gamma \lambda x^{-\beta}\right)\right]^{\alpha}$

where $(x, \gamma, \lambda, \alpha, \beta) \in \Re^{+}$. In cumulative distribution function (1), if $\gamma=1$ and $\beta=2$, the resulting distribution is called the Kumaraswamy-inverse Rayleigh distribution (Hussian [6]). The cumulative distribution function, probability density function and reliability function of Kumaraswamy-inverse Rayleigh distribution are given, respectively, by

$$
\begin{aligned}
& F(x)=1-\left[1-\exp \left(-\lambda x^{-2}\right)\right]^{\alpha} \\
& f(x)=2 \alpha \lambda x^{-3} \exp \left(-\lambda x^{-2}\right)\left[1-\exp \left(-\lambda x^{-2}\right)\right]^{\alpha-1} \\
& R(x)=\left[1-\exp \left(-\lambda x^{-2}\right)\right]^{\alpha}
\end{aligned}
$$


where $\alpha>0$ and $\lambda>0$ are shape and scale parameters respectively. In this article, we consider progressive firstfailure-censored samples from a Kumaraswamy-inverse Rayleigh distribution. we assumed that shape parameter $\beta$ is known and used Bayesian estimation method to estimate parameter $\alpha$ and reliability function of Kumaraswamyinverse Rayleigh distribution based on different symmetric and asymmetric loss functions.

\section{A progressive first failure censoring scheme}

In a life-testing experiment, Suppose that $n$ independent groups with $k$ items within each group are put in a life test. $R_{1}$ groups and the group in which the first failure is observed are randomly removed from the test as soon as the first failure $\left(X_{1: m: n: k}^{\mathbf{R}}=X_{1}\right)$ has occurred, $R_{2}$ groups and the group in which the second failure is observed are randomly removed from the test as soon as the second failure $\left(X_{2: m: n: k}^{\mathbf{R}}=X_{2}\right)$ has occurred, and finally $R_{m}(m \leq n)$ groups and the group in which the $m$-th failure is observed are randomly removed from the test as soon as the $m$-th failure $\left(X_{m: m: n: k}^{\mathbf{R}}=X_{m}\right)$ has occurred. Then $X_{1}<X_{2}<\cdots<X_{m}$ are called progressively first-failure-censored order statistics with the progressive censoring scheme $\mathbf{R}$. It is clear that $n=m+R_{1}+R_{2}+\cdots R_{m}$. If the failure times of the $n \times k$ items originally in the test are from a continuous population with distribution function $F(x)$ and probability density function $f(x)$, the joint probability density function for $X_{1}, X_{2}, \cdots, X_{m}$ is given by

$f\left(x_{1}, x_{2}, \cdots, x_{m}\right)=c k^{m} \prod_{i=1}^{m} f\left(x_{i}\right)\left(1-F\left(x_{i}\right)\right)^{k\left(R_{i}+1\right)-1} \quad 0<x_{1}<x_{2}<\cdots<x_{m}<\infty$

where $c=n\left(n-R_{1}-1\right)\left(n-R_{1}-R_{2}-2\right) \cdots\left(n-R_{1}-R_{2}-\cdots-R_{m-1}-m+1\right)$. Note that if $k=1$, the equation (5) reduces to the joint probability density function of progressively type II censored order statistics. If $\mathbf{R}=(0, \cdots, 0)$,equation (5) reduces to the joint probability density function of first-failure censored order statistics. If $k=1$ and $R=(0, \cdots, 0)$, then $n=m$ which corresponds to the complete sample, and if $k=1$ and $\mathbf{R}=$ $(0, \cdots, 0, n-m)$, then type II censored order statistics are obtained (See Wu and kus [1] or Wu and Huang [2]).

\section{Likelihood, prior and posterior}

Suppose $X_{1}<X_{2}<\cdots<X_{m}$ under the progressive censoring scheme $\mathbf{R}=\left(R_{1}, R_{2}, \cdots, R_{m}\right)$ are progressively first-failure-censored samples samples from the Kumaraswamy-inverse Rayleigh distribution which has probability density function (3) then the likelihood function for the observed $m$ samples can be written as follows:

$L(\alpha, \mathbf{X}) \propto \alpha^{m} e^{-\alpha u\left(x_{i}\right)}$

where $u\left(x_{i}\right)=-k \sum_{i=1}^{m}\left(R_{i}+1\right) \ln \left[1-\exp \left(-\lambda x_{i}^{-2}\right)\right]$. When parameter $\lambda$ is known, we consider the natural conjugate family of prior densities for parameter a as the following

$\pi(\alpha)=\frac{b^{a}}{\Gamma(a)} \alpha^{a-1} e^{-b \alpha}, \quad a, b>0$

From equations (6) and (7), the posterior distribution of $\alpha$ given the data $\mathbf{X}=\left(x_{1}, \cdots, x_{m}\right)$ is is thus

$\pi(\alpha \mid x)=\frac{\left(b+u\left(x_{i}\right)\right)^{m+a}}{\Gamma(m+a)} \alpha^{m+a-1} e^{-\alpha\left(b+u\left(x_{i}\right)\right)}$

\section{Bayesian Estimation}

\subsection{Bayes estimators under squared error loss function}

The Bayes estimator of $\alpha$, under the squared error loss function, is the posterior mean of the pdf given in Equation (8) and can be found as,

$\hat{\alpha}_{s . e}=\frac{m+a}{b+u\left(x_{i}\right)}$ 
Another problem of interest is that of estimating reliability function $R(t)$, with fixed $t>0$. The Bayes estimator of the reliability function $R(t)$, under squared error loss function, is given by

$\hat{S}_{s . e}=\left(\frac{b+u\left(x_{i}\right)}{b+u\left(x_{i}\right)-\ln w(t)}\right)^{(m+a)}$

where $w(t)=1-\exp \left(-\lambda t^{-2}\right)$

\subsection{Bayes estimators under minimum expected loss function}

In Bayesian estimation, widely used loss function is a quadratic loss function given by

$L(\lambda, \hat{\lambda})=w(\hat{\lambda}-\lambda)^{2}$

If $w=1$, it reduces to squared error loss function and for $w=\lambda^{-2}$,it becomes

$L(\lambda, \hat{\lambda})=\lambda^{-2}(\hat{\lambda}-\lambda)^{2}$

known as minimum expected loss function introduced by Rao Tummala and Sathe [4]. based on minimum expected loss function The Bayesian estimator of $\alpha$ is given by

$\hat{\alpha}_{m . e}=\frac{E\left(\lambda^{-1} \mid \mathbf{x}\right)}{E\left(\lambda^{-2} \mid \mathbf{x}\right)}$

Therefore we obtain Bayes estimator of the parameter $\alpha$ as the following form

$\hat{\alpha}_{m \cdot e}=\frac{m+a-2}{b+u\left(x_{i}\right)}$.

and based on minimum expected loss function The the Bayesian estimator of the reliability function $R(t)$ is:

$\hat{S}_{m . e}=\left(\frac{b+u\left(x_{i}\right)+2 \ln w(t)}{b+u\left(x_{i}\right)+\ln w(t)}\right)^{(m+a)}$

\subsection{Bayes estimators under weighted loss function}

The weighted loss function is defined as

$L(\hat{\alpha}-\alpha)=\frac{(\hat{\alpha}-\alpha)^{2}}{\alpha}, \quad \hat{\alpha}=\frac{1}{E(\alpha \mid x)}$

The Bayes estimator of parameter $\alpha$ under weighted loss function is given by

$\hat{\alpha}_{w . l}=\frac{m+a-1}{b+u\left(x_{i}\right)}$

Similarly, the Bayes estimator of the reliability function $R(t)$ under weighted loss function, is given by

$\hat{S}_{w . l}=\left(\frac{b+u\left(x_{i}\right)}{b+u\left(x_{i}\right)+\ln w(t)}\right)^{-(m+a)}$ 


\subsection{Bayes estimators under Linex loss function}

The LINEX loss function for $\alpha$ can be expressed as the following proportional

$L(\Delta) \propto \exp \gamma \Delta)-c \Delta-1 ; \quad \gamma \neq 0$

where $\Delta=(\hat{\alpha}-\alpha)$ and $\hat{\alpha}$ is an estimate of $\alpha$. The Bayes estimator of $\alpha$, under Linex loss function is given by (Zellner [7])

$\alpha_{\text {Linex }}=-\frac{1}{\gamma} \ln E_{\alpha}[\exp (-\gamma \alpha)]$

we obtain Bayesian estimator of the parameter $\alpha$, and reliability function $R(t)$, from (15) and (8) as the following forms

$\hat{\alpha}_{l i}=-\frac{m+a}{\gamma} \ln \left[\frac{b+u\left(x_{i}\right)}{b+u\left(x_{i}\right)+\gamma}\right]$

$\hat{S}_{l i}=-\frac{1}{\gamma} \ln \left[\sum_{j=0}^{\infty} \frac{(-\gamma)^{j}}{j !}\left(\frac{b+u\left(x_{i}\right)}{b+u\left(x_{i}\right)-j \ln w(t)}\right)^{(m+a)}\right]$

\section{Simulation study}

In order to compare the estimators of parameter and reliability function of Kumaraswamy-inverse Rayleigh distribution, Monte Carlo simulations were performed utilizing 2000 progressively first-failure-censored samples for each simulations. The mean square error (MSE) is used to compare the estimators. Using the algorithm presented in Balakrishnan and Sandhu [3], we generated progressively first failure censored samples from the Kumaraswamyinverse Rayleigh distribution with parameters $\alpha=\lambda=2$. The true value of $R(t)$, at $t=1$ is $R(1)=0.7476451$ and the prior parameters are $a=4, b=1$. The simulation results are summarized in tables 1 and 2 .

\section{Conclusions}

From Tables 1 and 2, it is clear that the Bayes estimates of parameter $\alpha$ and reliability function $S=R(t)$, under the LINEX loss function have the smallest estimated MSEs as compared with the estimates under squared error loss, minimum expected and weighted loss functions. It is immediate to note that MSEs decrease as sample size $n$ and $m$ increases.

\section{References}

[1] S. J. Wu and C. Kus, "On Estimation Based on Progressive First-Failure-Censored Sampling," Computational Statistics and Data Analysis, Vol. 53, No. 10, pp. 3659-3670, 2009.

[2] S.J. Wu, S. R. Huang, "Progressively first-failure censored reliability sampling plans with cost constraint," Computational Statistics 85 Data Analysis, Vol. 56, Issue 6, pp. 2018-2030, 2012.

[3] N. Balakrishnan, R. A. Sandhu, "A simple simulation algorithm for generating progressively type-II censored samples," The American Statistician 49, 229-230, 1995.

[4] V. M. Rao Tummala and P. T. Sathe, "Minimum expected loss estimators of reliability and parameters of certain lifetime distributions," IEEE Transactions on Reliability, 27,4, 283-285, 1978.

[5] M. Q. Shahbaz, S. Shahbaz, N. S. Butt, "The KumaraswamyInverse Weibull Distribution," Pakistan journal of statistics and operation research, 8(3): 479-489, 2012.

[6] M. A. Hussian and E. A. Amin, "Estimation and prediction for the Kumaraswamy-inverse Rayleigh distribution based on records ," International Journal of Advanced Statistics and Probability, 2,(1), 21-27, 2014.

[7] A. Zellner, "Bayes estimation and prediction using asymmetric loss functions," Journal of the American Statistical Association, 81, 446-451, 1986. 
Table 1: Estimated means and MSEs of various estimators of $\alpha$

\begin{tabular}{|c|c|c|c|c|c|c|c|}
\hline$k$ & $n$ & $m$ & $\mathbf{R}=\left(R_{1}, \ldots, R_{m}\right)$ & $\begin{array}{l}\hat{\alpha}_{s . e} \\
\operatorname{MSE}\left(\hat{\alpha}_{s . e}\right)\end{array}$ & $\begin{array}{l}\hat{\alpha}_{m . e} \\
\operatorname{MSE}\left(\hat{\alpha}_{m . e}\right)\end{array}$ & $\begin{array}{l}\hat{\alpha}_{w . l} \\
\operatorname{MSE}\left(\hat{\alpha}_{w . l}\right)\end{array}$ & $\begin{array}{l}\hat{\alpha}_{l i} \\
\operatorname{MSE}\left(\hat{\alpha}_{l i}\right)\end{array}$ \\
\hline 1 & 10 & 10 & $\begin{array}{l}(3,2,0,0,0) \\
(3,0 * 6) \\
(0, \ldots, 0)\end{array}$ & $\begin{array}{l}2.847335 \\
1.5246083 \\
2.642686 \\
1.0486233 \\
2.524530 \\
0.7685117\end{array}$ & $\begin{array}{l}2.214594 \\
0.5340129 \\
2.162198 \\
0.4517774 \\
2.163883 \\
0.3893408\end{array}$ & $\begin{array}{l}2.530964 \\
0.9192619 \\
2.402442 \\
0.6872304 \\
2.344207 \\
0.5438924\end{array}$ & $\begin{array}{l}2.173908 \\
0.3156078 \\
2.132708 \\
0.2913873 \\
2.137421 \\
0.2705353\end{array}$ \\
\hline 4 & 10 & 10 & $\begin{array}{l}(3,2,0,0,0) \\
(3,0 * 6) \\
(0, \ldots, 0)\end{array}$ & $\begin{array}{l}2.841031 \\
1.5425498 \\
2.639475 \\
1.0312187 \\
2.498519 \\
0.7196066 \\
\end{array}$ & $\begin{array}{l}2.209691 \\
0.5492249 \\
2.159570 \\
0.4420375 \\
2.141588 \\
0.3661507 \\
\end{array}$ & $\begin{array}{l}2.525361 \\
0.9359284 \\
2.399522 \\
0.6739081 \\
2.320053 \\
0.5086252 \\
\end{array}$ & $\begin{array}{l}2.168933 \\
0.3233388 \\
2.131072 \\
0.2851338 \\
2.119100 \\
0.2539655 \\
\end{array}$ \\
\hline 7 & 10 & 10 & $\begin{array}{l}(3,2,0,0,0) \\
(3,0 * 6) \\
(0, \ldots, 0)\end{array}$ & $\begin{array}{l}2.818108 \\
1.4931381 \\
2.647923 \\
1.0231730 \\
2.511919 \\
0.7573781\end{array}$ & $\begin{array}{l}2.191862 \\
0.5351818 \\
2.166483 \\
0.4316242 \\
2.153074 \\
0.3873378\end{array}$ & $\begin{array}{l}2.504985 \\
0.9059430 \\
2.407203 \\
0.6644658 \\
2.332496 \\
0.5376383\end{array}$ & $\begin{array}{l}2.155225 \\
0.3152019 \\
2.137603 \\
0.2778630 \\
2.128032 \\
0.2695625\end{array}$ \\
\hline 1 & 20 & 15 & $\begin{array}{l}(1,1, \ldots, 1) \\
\left(2,2,1,0^{*} 12\right) \\
(0, \ldots, 0)\end{array}$ & $\begin{array}{l}2.506057 \\
0.7390961 \\
2.355479 \\
0.4256996 \\
2.280428 \\
0.2996177\end{array}$ & $\begin{array}{l}2.148049 \\
0.3767774 \\
2.107534 \\
0.2511968 \\
2.090392 \\
0.1938536\end{array}$ & $\begin{array}{l}2.327053 \\
0.5234300 \\
2.231506 \\
0.3222498 \\
2.185410 \\
0.2373236\end{array}$ & $\begin{array}{l}2.124063 \\
0.2643520 \\
2.094320 \\
0.1954880 \\
2.081388 \\
0.1594534\end{array}$ \\
\hline 4 & 20 & 10 & $\begin{array}{l}(1,1, \ldots, 1) \\
\left(2,2,1,0^{*} 12\right) \\
(0, \ldots, 0)\end{array}$ & $\begin{array}{l}2.501284 \\
0.7016720 \\
2.352608 \\
0.4375895 \\
2.280359 \\
0.3083763\end{array}$ & $\begin{array}{l}2.143957 \\
0.3516201 \\
2.104965 \\
0.2617968 \\
2.090329 \\
0.2012343\end{array}$ & $\begin{array}{l}.322621 \\
0.4924276 \\
2.228787 \\
0.3334937 \\
2.185344 \\
0.2453785\end{array}$ & $\begin{array}{l}2.121721 \\
0.2477882 \\
2.091625 \\
0.2017639 \\
2.081086 \\
0.1651029\end{array}$ \\
\hline 7 & 20 & 10 & $\begin{array}{l}(1,1, \ldots, 1) \\
(2,2,1,0 * 12) \\
(0, \ldots, 0)\end{array}$ & $\begin{array}{l}.492238 \\
0.7410525 \\
2.353605 \\
0.4282152 \\
2.288809 \\
0.3283650\end{array}$ & $\begin{array}{l}2.136204 \\
0.3849834 \\
2.105857 \\
0.2539167 \\
2.098075 \\
0.2154483\end{array}$ & $\begin{array}{l}2.314221 \\
0.5287833 \\
2.229731 \\
0.3248814 \\
2.193442 \\
0.2623865\end{array}$ & $\begin{array}{l}2.113378 \\
0.2678319 \\
2.092719 \\
0.1967879 \\
2.087768 \\
0.1760092\end{array}$ \\
\hline
\end{tabular}


Table 2: Estimated means and MSEs of various estimators of reliability function $R(t)$

\begin{tabular}{|c|c|c|c|c|c|c|c|}
\hline$k$ & $n$ & $m$ & $\mathbf{R}=\left(R_{1}, \ldots, R_{m}\right)$ & $\begin{array}{l}\hat{S}_{s . e} \\
\operatorname{MSE}\left(\hat{S}_{s . e}\right)\end{array}$ & $\begin{array}{l}\hat{S}_{m \cdot e} \\
\operatorname{MSE}\left(\hat{S}_{m . e}\right)\end{array}$ & $\begin{array}{l}\hat{S}_{w \cdot l} \\
\operatorname{MSE}\left(\hat{S}_{w \cdot l}\right)\end{array}$ & $\begin{array}{l}\hat{S}_{l i} \\
\operatorname{MSE}\left(\hat{S}_{l i}\right)\end{array}$ \\
\hline 1 & 10 & 10 & $\begin{array}{l}(3,2,0,0,0) \\
(3,0 * 6) \\
(0, \ldots, 0)\end{array}$ & $\begin{array}{l}0.6727000 \\
0.04327193 \\
0.6899662 \\
0.03597317 \\
0.6995957 \\
0.03165453\end{array}$ & $\begin{array}{l}0.6458794 \\
0.05656659 \\
0.6706404 \\
0.04456047 \\
0.6856391 \\
0.03733389\end{array}$ & $\begin{array}{l}0.6598265 \\
0.04941108 \\
0.6806021 \\
0.04000882 \\
0.6927793 \\
0.03436473\end{array}$ & $\begin{array}{l}0.6858566 \\
0.04121359 \\
0.7070712 \\
0.03280836 \\
0.7188201 \\
0.02775752\end{array}$ \\
\hline 4 & 10 & 10 & $\begin{array}{l}(3,2,0,0,0) \\
(3,0 * 6) \\
(0, \ldots, 0)\end{array}$ & $\begin{array}{l}0.6734790 \\
0.04318256 \\
0.6901789 \\
0.03578172 \\
0.7020048 \\
0.03065364 \\
\end{array}$ & $\begin{array}{l}0.6467316 \\
0.05648195 \\
0.6709014 \\
0.04430803 \\
0.6882935 \\
0.03613722\end{array}$ & $\begin{array}{l}0.6606412 \\
0.04932247 \\
0.6808375 \\
0.03978939 \\
0.6953067 \\
0.03327118 \\
\end{array}$ & $\begin{array}{l}0.6869254 \\
0.04111850 \\
0.7072558 \\
0.03259602 \\
0.7215884 \\
0.02663498 \\
\end{array}$ \\
\hline 7 & 10 & 10 & $\begin{array}{l}(3,2,0,0,0) \\
(3,0 * 6) \\
(0, \ldots, 0)\end{array}$ & $\begin{array}{l}0.6755406 \\
0.04231564 \\
0.6892219 \\
0.03593251 \\
0.7008612 \\
0.03126968 \\
\end{array}$ & $\begin{array}{l}0.6491352 \\
0.05532197 \\
0.6698657 \\
0.04448401 \\
0.6870123 \\
0.03687913 \\
\end{array}$ & $\begin{array}{l}0.6628641 \\
0.04832181 \\
0.6798422 \\
0.03995239 \\
0.6940970 \\
0.03394666 \\
\end{array}$ & $\begin{array}{l}0.6893640 \\
0.04015553 \\
0.7059074 \\
0.03271775 \\
0.7204047 \\
0.02734437 \\
\end{array}$ \\
\hline 1 & 20 & 15 & $\begin{array}{l}(1,1, \ldots, 1) \\
\left(2,2,1,0^{*} 12\right) \\
(0, \ldots, 0)\end{array}$ & $\begin{array}{l}0.7013698 \\
0.03104680 \\
0.7143932 \\
0.02552196 \\
0.7210782 \\
0.02287209\end{array}$ & $\begin{array}{l}0.6875728 \\
0.03660561 \\
0.7053184 \\
0.02872237 \\
0.7143165 \\
0.02508610\end{array}$ & $\begin{array}{l}0.6946306 \\
0.03369999 \\
0.7099280 \\
0.02707156 \\
0.7177384 \\
0.02395220\end{array}$ & $\begin{array}{l}0.7210210 \\
0.02711539 \\
0.7366496 \\
0.02086443 \\
0.7447080 \\
0.01787462\end{array}$ \\
\hline 4 & 20 & 10 & $\begin{array}{l}(1,1, \ldots, 1) \\
\left(2,2,1,0^{*} 12\right) \\
(0, \ldots, 0)\end{array}$ & $\begin{array}{l}0.7016120 \\
0.03069341 \\
0.7147744 \\
0.02549235 \\
0.7211487 \\
0.02293052\end{array}$ & $\begin{array}{l}0.6878760 \\
0.03616738 \\
0.7057150 \\
0.02869704 \\
0.7143839 \\
0.02515304\end{array}$ & $\begin{array}{l}0.6949017 \\
0.03330689 \\
0.7103168 \\
0.02704381 \\
0.7178074 \\
0.02401471\end{array}$ & $\begin{array}{l}0.7210937 \\
0.02669613 \\
0.7371668 \\
0.02083327 \\
0.7448684 \\
0.01794961\end{array}$ \\
\hline 7 & 20 & 10 & $\begin{array}{l}(1,1, \ldots, 1) \\
(2,2,1,0 * 12) \\
(0, \ldots, 0)\end{array}$ & $\begin{array}{l}0.7028376 \\
0.03065798 \\
0.7146062 \\
0.02547412 \\
0.7203858 \\
0.02328193\end{array}$ & $\begin{array}{l}0.6891592 \\
0.03615785 \\
0.7055433 \\
0.02867081 \\
0.7135744 \\
0.02554478\end{array}$ & $\begin{array}{l}0.6961560 \\
0.03328276 \\
0.7101468 \\
0.02702186 \\
0.7170217 \\
0.02438564\end{array}$ & $\begin{array}{l}0.7228813 \\
0.02670291 \\
0.7369199 \\
0.02081412 \\
0.7440087 \\
0.01832605\end{array}$ \\
\hline
\end{tabular}

